\title{
Applications of Double Framed T-Soft Fuzzy Sets in BCK/BCI-Algebras
}

\author{
Muhammad Bilal Khan ${ }^{\mathrm{a},{ }^{*}}$ and Tahir Mahmood ${ }^{\mathrm{b},{ }^{*}}$ \\ *Department of Mathematics and Statistics, International Islamic University Islamabad, \\ Pakistan
}

\section{abilal.msma360@iiu.edu.pk,}

\begin{abstract}
s:
The aim of this article is introduced the concept of double framed T-soft fuzzy set (DFT-soft fuzzy set) which is the combination of soft set and fuzzy set. We also defined the notions and apply this concept in BCK/BCI-algebras. By using example, we also discussed the concept of double framed T-soft fuzzy algebra (DFT-soft fuzzy algebra) and double framed B-soft fuzzy algebra (DFB-soft fuzzy algebra) and also investigated their properties. Each double framed T-soft fuzzy algebra is double framed B-soft fuzzy algebra but by using example, we proved that converse may or may not be possible.
\end{abstract}

Keywords: double framed T-soft fuzzy set; double framed T-soft fuzzy algebra; double framed B-soft fuzzy algebra

\section{Introduction}

Traditionally mathematics uses crisp set theory [1] to explain the properties of any substance and this set theory consists of two possibilities that are either false or true. But with the passage of time, the vague concepts became a virus in different fields of our life like pharmacology, engineering, medical application and economics but classical mathematical tools are failed to solve these problems. Then in 1965, Zadeh [2] introduced most successful theory which is known as fuzzy set theory which dealt with the vague concepts. This theory is used openly in different fields like pharmacology, engineering, medical application and economics, among others. We know that fuzzy set theory is strongly based on membership function. We can determine the membership grade of an element of a set with the help of membership function. The fuzzy set theory has become very popular. But there was a difficulty to set a membership grade of a membership function. To solve this problem Moldstov [3] presented the theory which is known as soft set theory. This theory is used successfully in different areas such as game theory, Riemann-integration, smoothness of function, Perron-integration, etc. In 2002 Maji et al. [4] introduced the application of soft set theory in decision making problems. Also Maji [5] in 2003 studied the theoretical work on soft set theory to polish this concept so that readers could easily understand and contributed their role to extend the scope of this theory in different fields of life. After theoretical discussion, now we discussed about the contributions of those researchers whose applied this concept in different fields of algebra like Cagman et al. [6] studied the soft set and soft groups and also introduced the notation of soft groups. They also defined the relation between fuzzy set, rough set and soft set and discuss the properties. Ali et.al [7] introduced some new operations on soft sets. 
2|P a g e

For the study of $\mathrm{BCK} / \mathrm{BCI}$ algebras we refer the readers to books $[8,9]$. Jun et al. [10] worked on intersectional soft sets and defined its applications in BCK/BCI algebras. They also defined the notations of intersectional soft sets. Also Jun [11], studied the union soft sets and defined its application in BCK/BCI algebras. Further, Jun and Park [12] elaborated applications of soft ideal theory in BCK/BCI algebras. Jun and Ahn [13] studied the applications of double framed soft sets. They also introduced the notations of double framed soft algebras and discussed their properties by giving several examples. Naz [14] introduced some operations on double framed double soft sets. Hadipour [15] applied the concept of double framed soft set in BF-algebras and introduced the notations of double framed BFalgebras. He also investigated its properties. Cho et al. [16] studied the concept of double framed soft near ring. Shabir and Samreena [17], worked on double framed soft topological spaces and defined its notation. For further information, we refer to reader papers [18-29] regarding soft algebraic structures.

From inspiring above literature, In section 2, we discussed basic definitions related to soft set, double framed soft set and $\mathrm{BCK} / \mathrm{BCI}$ algebras. In section 3. our basic purpose to combine the concept of soft set and fuzzy set in pair form and then this concept is used in BCK/BCI algebras and introduced its notations. We investigated the properties of double framed T-soft fuzzy algebra and double framed B-soft fuzzy algebras. We elaborated that each double framed T-soft fuzzy algebra is also double framed B-soft fuzzy algebra but converse is may or may not possible.

\section{Preliminaries}

Let $X$ be a non-empty set. Then $A=\left\{\left\langle x, \mu_{A}(x)\right\rangle \mid x \in X\right\}$ is called fuzzy set, where $\mu_{A}$ is a membership function which map each element of $X$ in $[0,1]$. Here we say that $A$ is fuzzy subset of $X$. The set of all fuzzy subsets of a set $X$ is denoted by $F P(X)$. Two fuzzy sets $A$ and $B$ are equal if and only if $\mu_{A}(x)=\mu_{B}(x)$ for all $x \in X$. Where $\mu_{A}$ and $\mu_{B}$ are membership functions which map each element of $X$ in $[0,1]$. The complement of a fuzzy set $A$ is denoted by $A^{c}$ and is defined by,

$$
\mu_{A^{c}}=1-\mu_{A}
$$

Where $\mu_{A}$ is a membership function which map each element of $X$ in $[0,1]$. Let $X$ be a nonempty set. The union of two fuzzy sets $A$ and $B$ with membership functions $\mu_{A}(x)$ and $\mu_{B}(x)$ respectively is denoted and defined by,

$$
\mu_{A \cup B}(x)=\max \left\{\mu_{A}(x), \mu_{B}(x)\right\} \text { for all } x \in X
$$


3| P a g e

Here $\mu_{A}$ and $\mu_{B}$ are membership functions which map each element of $X$ in $[0,1]$. In abbreviated form $\mu_{C}=\mu_{A} \vee \mu_{B}$, where $C=A \cup B$. The intersection of two fuzzy sets $A$ and $B$ with membership functions $\mu_{A}(x)$ and $\mu_{B}(x)$ respectively is denoted and defined by,

$$
\mu_{A \cap B}(x)=\min \left\{\mu_{A}(x), \mu_{B}(x)\right\} \text { for all } x \in X
$$

Here $\mu_{A}$ and $\mu_{B}$ are membership functions which map each element of $X$ in $[0,1]$. In abbreviated form $\mu_{C}=\mu_{A} \wedge \mu_{B}$, where $C=A \cap B$.

Cagman [6] introduced the new definition of soft set in this way,

A pair $(\Gamma, E)$ is indicated to be soft set over if and only if $\Gamma$ is a mapping from $E$ to the all subset of $U$ e.g

$$
(\Gamma, E)=\{(e, \Gamma(e)) \mid e \in E \text { and } \Gamma(e) \in P(U)\}
$$

Where $P(U)$ is power set of $U$ and $\Gamma: E \rightarrow P(U)$. The function $\Gamma$ is an approximation function of the soft set $(\Gamma, E)$. It is easy to see that soft set is parameterized family of subsets of $U$.

The set of all soft sets over $U$ is denoted by $S(U)$.

BCK/BCI-algebras defined by K. Iseki, in such a way,

A non-empty set $X$ under binary operation " * " with $\theta$ is called a BCI-algebra of type $(2, \theta)$ is denoted by $(X, *, \theta)$ and defined as

(1) $((u * v) *(u * w)) *(w * v)=\theta$ for all $u, v, w \in X$,

(2) $(u *(u * v)) * v=\theta$ for all $u, v \in X$,

(3) $u * u=\theta$ for all $u \in X$,

(4) $u * v=\theta, v * u=\theta$ impies $u=v$ for all $u, v \in X$.

If a BCI-algebra $X$ satisfies, $\theta * u=\theta$ for all $u \in X$ then $X$ is called BCK-algebra. For any BCI/BCK-algebra the following hold, $u * \theta=u$ for all $u \in X$. 
4| P a g e

Jun at al. [13] introduced the double framed soft set in such a way,

A double framed pair $((F,(\mathfrak{5}), A)$ over $U$ is said to double framed soft set, in pair form $(F,(\mathfrak{5})$ both are soft sets over $U$. A double framed pair $((F, \mathfrak{5}), A)$ over $U$ is said to be double framed soft algebra if it satisfies

$F(u * v) \geq F(u) \cap F(v)$ and $\mathfrak{G}(u * v) \subseteq \mathfrak{G}(u) \cup \mathfrak{G}(v)$ for all $u, v \in A$

\section{Double framed T-soft fuzzy algebra and double framed B-soft fuzzy algebra}

In this section, We elaborate the double framed T-soft fuzzy set, double framed T-soft fuzzy algebra, double framed B-soft fuzzy algebra and investigate their properties. In all examples, all defined BCI/BCK algebras are taken from [13] except 3.12. Example.

Note that we take $\delta=X$, which is $\mathrm{BCI} / \mathrm{BCK}$ algebras, where $\delta$ is a set of parameters, and for sub algebras, we use $A, B, C$ of $\delta$, otherwise mentioned.

\subsection{Definition}

Let $(F, A)$ is a soft set over $U$ and $f: A \rightarrow I($ where $I=[0,1])$ is a fuzzy set then double framed pair $((F, f), A)$ over $(U,[0,1])$ is called double framed T-soft fuzzy set (DFT-soft fuzzy set ).

\subsection{Definition}

Let $((F, f), A)$ and $(((5, g), B)$ be two "DFT-soft fuzzy set" over $(U,[0,1])$ then $((F, f), A)$ is said to be double framed T-soft fuzzy subset of $((\mathfrak{5}, g), B)$ if

(1) $A \subseteq B$,

(2) $F(a) \subseteq \mathfrak{G}(a)$ and $f(a) \geq g(a)$ for all $a \in A$.

We can write $((F, f), A) \widetilde{\subset}(((\mathfrak{H}, g), B)$. In case $((F, f), A)$ is super set of $((\mathfrak{G}, g), B)$.

\subsection{Definition}

Let $((F, f), A)$ and $(((5, g), B)$ be two "DFT-soft fuzzy set" over $(U,[0,1])$ then $((F, f), A)$ is said to be double framed T-soft fuzzy twisted subset of $((\mathfrak{G}, g), B)$ if

(1) $A \subseteq B$,

(2) $F(a) \supseteq \mathfrak{b}(a)$ and $f(a) \leq g(a)$ for all $a \in A$.

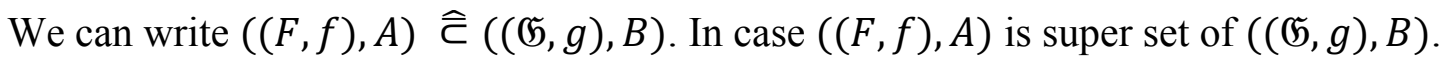

\subsection{Definition}


$\mathbf{5} \mid \mathrm{P}$ a g e

A "DFT-soft fuzzy set" $((F, f), A)$ over $(U,[0,1])$ is said to be a "DFT-soft fuzzy algebra" over $(U,[0,1])$ if it satisfies

$F(x * y) \subseteq F(x) \cup F(y)$ and $f(x * y) \geq f(x) \wedge f(y)$ for all $x, y \in A$.

\subsection{Example}

Suppose $\delta=\left\{p_{\theta}, p_{1}, p_{2}, p_{3}\right\}$ be the set of parameter, (where " $p_{\theta}=$ beautiful", " $p_{1}=$ cheap", " $p_{2}=$ in good location", $p_{3}=$ in green surroundings) with the following binary operation

\begin{tabular}{|c|c|c|c|c|}
\hline$*$ & $p_{\theta}$ & $p_{1}$ & $p_{2}$ & $p_{3}$ \\
\hline$p_{\theta}$ & $p_{\theta}$ & $p_{\theta}$ & $p_{\theta}$ & $p_{\theta}$ \\
\hline$p_{1}$ & $p_{1}$ & $p_{\theta}$ & $p_{1}$ & $p_{1}$ \\
\hline$p_{2}$ & $p_{2}$ & $p_{2}$ & $p_{\theta}$ & $p_{2}$ \\
\hline$p_{3}$ & $p_{3}$ & $p_{3}$ & $p_{3}$ & $p_{\theta}$ \\
\hline
\end{tabular}

$\left(\delta, *, p_{\theta}\right)$ is BCK-algebra And $U=\left\{h_{1}, h_{2}, h_{3}, h_{4}, h_{5}\right\}$ is the initial universe set that containing five houses.

Now we define a "DFT-soft fuzzy set" as follows

$$
F: \delta \rightarrow P(U), y \mapsto \begin{cases}U & \text { if } y=p_{\theta} \\ \left\{h_{1}, h_{2}, h_{5}\right\} & \text { if } y=p_{1} \\ \left\{h_{1}, h_{2}, h_{3}, h_{5}\right\} & \text { if } y=p_{2} \\ \left\{h_{1}, h_{2}\right\} & \text { if } y=p_{3}\end{cases}
$$

Define $f: \delta \rightarrow\left[\begin{array}{ll}0 & 1]\end{array}\right]$ such that $f\left(p_{\theta}\right)=0.7, f(y)=.5$. Then $((F, f), \delta)$ is "DFT-soft fuzzy $\operatorname{set}^{\prime \prime}(U,[0,1])$.

\subsection{Example}

Consider the initial universe set $U=\left\{h_{1}, h_{2}, h_{3}, h_{4}, h_{5}\right\}$ and a set of parameters $\delta=\left\{p_{\theta}\right.$, $\left.p_{1}, p_{2}, p_{3}\right\}$ which is defined in above equation under the binary operation "*" such that 


\begin{tabular}{|c|c|c|c|c|}
\hline$*$ & $p_{\theta}$ & $p_{1}$ & $p_{2}$ & $p_{3}$ \\
\hline$p_{\theta}$ & $p_{\theta}$ & $p_{\theta}$ & $p_{\theta}$ & $p_{\theta}$ \\
\hline$p_{1}$ & $p_{1}$ & $p_{\theta}$ & $p_{1}$ & $p_{1}$ \\
\hline$p_{2}$ & $p_{2}$ & $p_{2}$ & $p_{\theta}$ & $p_{2}$ \\
\hline$p_{3}$ & $p_{3}$ & $p_{3}$ & $p_{3}$ & $p_{\theta}$ \\
\hline
\end{tabular}

And $A=\left\{p_{\theta}, p_{2}, p_{3}\right\}$ is a subalgebra of $\delta$. Now consider $((F, f), \delta)$ "DFT-soft fuzzy set" as follows

$$
F: \delta \rightarrow P(U), y \mapsto \begin{cases}\left\{h_{2}\right\} & \text { if } y=p_{\theta} \\ \left\{h_{1}, h_{2}, h_{3}\right\} & \text { if } y=p_{2} \\ \left\{h_{1}, h_{2}\right\} & \text { if } y=p_{3}\end{cases}
$$

Define $f: \delta \rightarrow\left[\begin{array}{ll}0 & 1\end{array}\right]$ such that $f\left(p_{\theta}\right)=0.8, f\left(p_{2}\right)=0.6$ and $f\left(p_{3}\right)=0.5$. Then $((F, f), A)$ is "DFT-soft fuzzy set" $(U,[0,1])$.

\subsection{Example}

Let $\left(\delta, *, p_{\theta}\right)$ is BCI-algebra, where $\delta=\left\{p_{\theta}, p_{1}, p_{2}, p_{3}, p_{4}, p_{5}, p_{6}, p_{7}\right\}$, defined by

\begin{tabular}{|c|c|c|c|c|c|c|c|c|}
\hline$*$ & $p_{\theta}$ & $p_{1}$ & $p_{2}$ & $p_{3}$ & $p_{4}$ & $p_{5}$ & $p_{6}$ & $p_{6}$ \\
\hline$p_{\theta}$ & $p_{\theta}$ & $p_{\theta}$ & $p_{\theta}$ & $p_{\theta}$ & $p_{4}$ & $p_{4}$ & $p_{4}$ & $p_{4}$ \\
\hline$p_{1}$ & $p_{1}$ & $p_{\theta}$ & $p_{\theta}$ & $p_{\theta}$ & $p_{5}$ & $p_{4}$ & $p_{4}$ & $p_{4}$ \\
\hline$p_{2}$ & $p_{2}$ & $p_{2}$ & $p_{\theta}$ & $p_{\theta}$ & $p_{6}$ & $p_{6}$ & $p_{4}$ & $p_{4}$ \\
\hline$p_{3}$ & $p_{3}$ & $p_{2}$ & $p_{1}$ & $p_{\theta}$ & $p_{7}$ & $p_{6}$ & $p_{5}$ & $p_{4}$ \\
\hline$p_{4}$ & $p_{4}$ & $p_{4}$ & $p_{4}$ & $p_{4}$ & $p_{\theta}$ & $p_{\theta}$ & $p_{\theta}$ & $p_{\theta}$ \\
\hline$p_{5}$ & $p_{5}$ & $p_{4}$ & $p_{4}$ & $p_{4}$ & $p_{1}$ & $p_{\theta}$ & $p_{\theta}$ & $p_{\theta}$ \\
\hline$p_{6}$ & $p_{6}$ & $p_{6}$ & $p_{4}$ & $p_{4}$ & $p_{2}$ & $p_{2}$ & $p_{\theta}$ & $p_{\theta}$ \\
\hline$p_{7}$ & $p_{7}$ & $p_{6}$ & $p_{5}$ & $p_{4}$ & $p_{3}$ & $p_{2}$ & $p_{1}$ & $p_{\theta}$ \\
\hline
\end{tabular}


7| P a g e

Now consider a "DFT-soft fuzzy set" $((F, f), \delta)$, as follows

$F: \delta \rightarrow P(U), y \mapsto \begin{cases}\left\{h_{1}, h_{3}, h_{5}, h_{7}\right\} & \text { if } y \in\left\{p_{\theta}, p_{1}, p_{2}, p_{3}\right\} \\ \left\{h_{1}, h_{2}, h_{4}\right\} & \text { if } y \in\left\{p_{4}, p_{5}, p_{6}, p_{7}\right\}\end{cases}$

where $U=\left\{h_{1}, h_{2}, h_{3}, h_{4}, h_{5}, h_{6} h_{7}\right\}$.

Define $f: \delta \rightarrow\left[\begin{array}{ll}0 & 1\end{array}\right]$ such that $f\left(p_{\theta}\right)=0.8, f\left(p_{1}\right)=0.7, f\left(p_{2}\right)=0.6$ and $f\left(p_{3}\right)=0.5$, $f\left(p_{4}\right)=1=f\left(p_{5}\right), f\left(p_{6}\right)=0.9=f\left(p_{7}\right)$.

Then we have

$F\left(p_{6} * p_{5}\right)=F\left(p_{2}\right)=\left\{h_{1}, h_{3}, h_{5}, h_{7}\right\} \nsubseteq\left\{h_{1}, h_{2}, h_{4}\right\}=F\left(p_{6}\right) \cup F\left(p_{5}\right)$

And

$f\left(p_{6} * p_{5}\right)=f\left(p_{2}\right)=.6 \geq .9=.9 \wedge .9=f\left(p_{6}\right) \wedge f\left(p_{5}\right)$

Implies $((F, f), \delta)$ is not a "DFT-soft fuzzy algebra" over $(U,[0,1])$.

\subsection{Lemma}

Every "DFT-soft fuzzy algebra" $((F, f), \delta)$ over $(U,[0,1])$ satisfies the following condition $F(\theta) \subseteq F(x)$ and $f(\theta) \geq f(x)$ for all $x \in \delta$.

\section{Proof}

Since $F(x * x) \subseteq F(x) \cup F(x)$ for all $x \in \delta$ implies $F(x * x)=F(\theta) \subseteq F(x)$ because $x *$ $x=\theta$.

Now $f(x * x) \geq f(x) \wedge f(x)$ for all $x \in \delta$. Since $x * x=\theta$ so $f(x * x)=f(\theta) \geq f(x)$.

Hence, proof is complete.

\subsection{Theorem}

For a "DFT-soft fuzzy algebra" $((F, f), \delta)$ over $(U,[0,1])$, the following are equivalent;

(1) $F(\theta)=F(x)$ and $f(\theta)=f(x)$ for all $x \in \delta$,

(2) $F(x * y) \subseteq F(y)$ and $f(x * y) \geq f(y)$ for all $x, y \in \delta$.

Proof

$(1) \Rightarrow(2)$, We suppose that $(1)$ is valid. Now by definition of $((F, f), \delta)$, we have

(for all $x \in \delta$ ) $F(x * y) \subseteq F(x) \cup F(y)=F(\theta) \cup F(y)=F(y)$ because $F(\theta)=$ $F(x)$ for all $x \in \delta$, 
8| P a g e

Now (for all $x \in \delta) f(x * y) \geq f(x) \wedge f(y)=f(\theta) \wedge f(y)=f(y)$ because $f(\theta)=$ $f(x)$ for all $x \in \delta$.

(2) $\Rightarrow(1)$, Assume that (2) is valid. Then for $y=\theta$ such that $F(x * \theta) \subseteq F(\theta)$ and $f(x *$ $\theta) \geq f(\theta)$ implies $F(x) \subseteq F(\theta)$ and $f(x) \geq f(\theta)$ because $x * \theta=x$, then by 3.8. Lemma we have, $F(\theta) \subseteq F(x)$ and $f(\theta) \geq f(x)$ for all $x \in \delta$, so $F(x)=F(\theta)$ and $f(x)=f(\theta)$.

\subsection{Proposition}

In a BCI-algebra $X$, every "DFT-soft fuzzy algebra" $((F, f), A)$ over $(U,[0,1])$ satisfies the following condition

For all $u, v \in A\left\{\begin{array}{l}F(u *(\theta * v)) \subseteq F(u) \cup F(v) \\ f(u *(\theta * v)) \geq f(u) \wedge f(v)\end{array}\right.$

Proof: Let $u, v \in A$, we have

$F(u *(\theta * v)) \subseteq F(u) \cup F(\theta * v) \subseteq F(u) \cup(F(\theta) \cup F(v)) \because((F, f), A)$ is double framed soft fuzzy algebra implies $F(u *(\theta * v)) \subseteq F(u) \cup F(v)$ by 3.8. Lemma.

Now $f(u *(\theta * v)) \geq f(u) \wedge f(\theta * v) \geq f(u) \wedge(f(\theta) \wedge f(v)) \because((F, f), A)$ is double framed soft fuzzy algebra implies $f(u *(\theta * v)) \geq f(u) \wedge f(v)$ by 3.8. Lemma.

Hence proved.

\subsection{Definition}

A "DFT-soft fuzzy set" $((F, f), \delta)$ over $(U,[0,1])$ is said to be a "DFB-soft fuzzy algebra" over $(U,[0,1])$ if it satisfies

$F(x * y) \subseteq F(x) \cup F(y)$ and $f(x * y) \geq f(x) . f(y)$ for all $x, y \in \delta$

\subsection{Example}

Let $\delta=\left\{\xi_{\theta}, \xi_{1}, \xi_{2}, \xi_{3}\right\}$ be a set with binary operation " * " defined by

\begin{tabular}{|c|c|c|c|c|}
\hline$*$ & $\xi_{\theta}$ & $\xi_{1}$ & $\xi_{2}$ & $\xi_{3}$ \\
\hline$\xi_{\theta}$ & $\xi_{\theta}$ & $\xi_{1}$ & $\xi_{2}$ & $\xi_{3}$ \\
\hline$\xi_{1}$ & $\xi_{1}$ & $\xi_{\theta}$ & $\xi_{3}$ & $\xi_{2}$ \\
\hline$\xi_{2}$ & $\xi_{2}$ & $\xi_{3}$ & $\xi_{\theta}$ & $\xi_{1}$ \\
\hline$\xi_{3}$ & $\xi_{3}$ & $\xi_{2}$ & $\xi_{1}$ & $\xi_{\theta}$ \\
\hline
\end{tabular}


9| P a g e

Then $\left(\delta, *, \xi_{\theta}\right)$ is a BCl-algebra. Define

$F: \delta \rightarrow P(U), y \mapsto\left\{\begin{array}{l}\left\{h_{1}, h_{3}, h_{5}\right\} \text { if } y=\xi_{\theta} \\ U \text { if } y \in\left\{\xi_{1}, \xi_{2}, \xi_{3}\right\}\end{array}\right.$ where $U=\left\{h_{1}, h_{2}, h_{3}, h_{4}, h_{5}, h_{6} h_{7}\right\}$

And

$f: \delta \rightarrow\left[\begin{array}{ll}0 & 1\end{array}\right]$ such that $f\left(\xi_{\theta}\right)=0.6, f(y)=0.7$ for all $y \in\left\{\xi_{1}, \xi_{2}, \xi_{3}\right\}$

It is routine to verify $((F, f), \delta)$ is a "DFB-soft fuzzy algebra" over $(U,[0,1])$.

\subsection{Theorem}

Let $((F, f), \delta)$ be a "DFT-soft fuzzy set" over $(U,[0,1])$. Then prove that "DFT-soft fuzzy algebra" of $\delta$ over $(U,[0,1])$ is also "DFB-soft fuzzy algebra" of $\delta$ over $(U,[0,1])$.

Proof

Let $((F, f), \delta)$ be a "DFT-soft fuzzy algebra" over $(U,[0,1])$ to prove $((F, f), \delta)$ be a "DFBsoft fuzzy algebra" over $(U,[0,1])$.

As $((F, f), \delta)$ be a "DFT-soft fuzzy algebra" over $(U,[0,1])$ then for any $u, v \in \delta$, we have $F(u * v) \subseteq F(u) \cup F(v)$ is obvious,

$f(u * v) \geq f(u) \wedge f(v) \geq f(u) . f(v)$ implies $f(u * v) \geq f(u) . f(v)$.

Hence, $((F, f), \delta)$ is "DFB-soft fuzzy algebra" over $(U,[0,1])$.

Note that converse of above theorem may or may not be true.

3.12. Example represented that $((F, f), \delta)$ is a "DFB-soft fuzzy algebra" of $\delta$ over $(U,[0,1])$ but not a "DFT-soft fuzzy algebra" of $\delta$ over $(U,[0,1])$ because

$f\left(\xi_{1} * \xi_{1}\right)=f\left(\xi_{\theta}\right)=0.6 \geq 0.7=0.7 \wedge 0.7=f\left(\xi_{1}\right) \wedge f\left(\xi_{1}\right)$.

\subsection{Lemma}

Each "DFB-soft fuzzy algebra" $((F, f), \delta)$ over $(U,[0,1])$ satisfies the following condition $F(\theta) \subseteq F(x)$ and $f(\theta) \geq(f(x))^{2}$ for all $x \in \delta$.

\section{Proof}

Since $F(x * x) \subseteq F(x) \cup F(x)$ for all $x \in \delta$ implies $F(x * x)=F(\theta) \subseteq F(x)$ because $x *$ $x=\theta$.

Now $f(x * x) \geq f(x) . f(x)$ for all $x \in \delta$, since $x * x=\theta$ so $f(x * x)=f(\theta) \geq(f(x))^{2}$.

Hence, proof is complete. 
$10 \mid \mathrm{P}$ a g e

\subsection{Proposition}

In a BCI-algebra $X$, every "DFB-soft fuzzy algebra" $((F, f), A)$ over $(U,[0,1])$ satisfies the following condition

For all $u, v \in A\left\{\begin{array}{l}F(u *(\theta * v)) \subseteq F(u) \cup F(v) \\ f(u *(\theta * v)) \geq f(u) . f(v)^{3}\end{array}\right.$

Proof: Let $u, v \in A$, we have

$F(u *(\theta * v)) \subseteq F(u) \cup F(\theta * v) \subseteq F(u) \cup(F .(\theta) \cup F(v)) \because((F, f), A)$ is double framed soft fuzzy algebra over $(U,[0,1])$ implies $F(u *(\theta * v)) \subseteq F(u) \cup F(v)$, by 3.14. Lemma.

Now $f(u *(\theta * v)) \geq f(u) . f(\theta * v) \geq f(u) .(f(\theta) . f(v)) \because((F, f), A)$ is double framed soft fuzzy algebra over $(U,[0,1])$,

Implies $f(u *(\theta * v)) \geq f(u) \wedge f(v) \geq f(u) .\left(f(v)^{2} . f(v)\right)$, by 3.14. Lemma,

Implies $f(u *(\theta * v)) \geq f(u) \cdot f(v)^{3}$.

Hence, proof is complete.

\subsection{Theorem}

Let $((F, f), A)$ over $(U,[0,1])$ be a double framed T-soft fuzzy subset of a double framed soft fuzzy set $((\mathfrak{G}, g), B)$ over $(U,[0,1])$ where $F(p)$ and $(\mathfrak{G}(p)$ both are identically approximations and $f(p)$ and $g(p)$ both are identical approximations for all $p \in A$. If $((\mathfrak{F}, g), B)$ is a "DFT-soft fuzzy algebra" over $(U,[0,1])$ then $((F, f), A)$ is both "DFT-soft fuzzy algebra" over $(U,[0,1])$ and "DFB-soft fuzzy algebra" over $(U,[0,1])$.

Proof

Let $u, v \in A$ then , $v \in B \because A \subseteq B$.

As $((\mathfrak{5}, g), B)$ is a "DFT-soft fuzzy algebra" over $(U,[0,1])$ so

$\mathfrak{b}(u) \cup \mathfrak{b}(v)=F(u) \cup F(v) \supseteq F(u * v)=\mathfrak{5}(u * v)$ implies $F(u) \cup F(v) \supseteq F(u * v)$.

$\because F(p)$ and $\mathfrak{G}(p)$ are identical approximations.

$g(u) \wedge g(v)=f(u) \wedge f(v) \leq f(u * v)=g(u * v)$ implies $f(u) \wedge f(v) \leq f(u * v)$.

$\because f(p)$ and $g(p)$ are identical approximations.

Hence $((F, f), A)$ is a "DFT-soft fuzzy algebra" over $(U,[0,1])$.

Now, $f(u) \cdot f(v)=g(u) \cdot g(v) \leq f(u) \wedge f(v)=g(u) \wedge g(v)=f(u) \wedge f(v) \leq f(u * v)=$ $g(u * v)$ implies $f(u) . f(v) \leq f(u * v) . \because f(p)$ and $g(p)$ are identical approximations.

Hence $((F, f), A)$ is a double frame B-soft fuzzy algebra over $(U,[0,1])$. 
11 | P a g e

\subsection{Theorem}

Let $((F, f), A)$ over $(U,[0,1])$ be a double framed B-soft fuzzy subset of a double framed soft fuzzy set $((\mathfrak{G}, g), B)$ over $(U,[0,1])$ where $F(p)$ and $\mathfrak{G}(p)$ both are identically approximations and $f(p)$ and $g(p)$ both are identical approximations for all $p \in A$. If $((\mathfrak{F}, g), B)$ is a "DFB-soft fuzzy algebra" over $(U,[0,1])$ then $((F, f), A)$ is a "DFB-soft fuzzy algebra" over $(U,[0,1])$.

Proof

Let $u, v \in A$ then $u, v \in B \because A \subseteq B$.

As $(((5, g), B)$ is a "DFT-soft fuzzy algebra" over $(U,[0,1])$ so

$\mathfrak{b}(u) \cup \mathfrak{5}(v)=F(u) \cup F(v) \supseteq F(u * v)=\mathfrak{G}(u * v)$ implies $F(u) \cup F(v) \supseteq F(u * v)$.

$\because F(p)$ and $\mathfrak{5}(p)$ are identical approximations.

$g(u) \cdot g(v)=f(u) \cdot f(v) \leq f(u * v)=g(u * v)$ implies $f(u) \cdot f(v) \leq f(u * v) . \because f(p)$ and $g(p)$ are identical approximations.

Hence $((F, f), A)$ is a double frame B-soft algebra over $(U,[0,1])$.

\section{Remark}

(1) Converse of above theorem is may or may not be possible.

(2) $((F, f), A)$ is may or may not be "DFT-soft fuzzy algebra" over $(U,[0,1])$ because 3.12 . example represented that each "DFB-soft fuzzy algebra" is not "DFT-soft fuzzy algebra".

\subsection{Definition}

Let $V_{(F, f)}$ and $B_{(\mathfrak{G}, g)}$ are two "DFT-soft fuzzy set" over $(U,[0,1])$. Then extended uni-int "DFT-soft fuzzy set" of $V_{(F, f)}$ and $B_{(\mathfrak{G}, g)}$ is defined as a "DFT-SS" $(V \cup B)_{(F \widetilde{\cup} \mathfrak{G}, f \bar{n} g)}$,

Where $F \widetilde{\cup} \mathfrak{5}:(V \cup B) \rightarrow P(U)$ defined by

$$
p \rightarrow\left\{\begin{array}{c}
F(p) \text { if } p \in V-B \\
\mathfrak{G}(p) \text { if } p \in B-V \\
F(p) \cup \mathfrak{G}(p) \text { if } p \in V \cap B
\end{array}\right.
$$

and $f \bar{\cap} g:(V \cup B) \rightarrow[0,1]$ defined by

$p \rightarrow\left\{\begin{array}{c}f(p) \text { if } p \in V-B \\ g(p) \text { if } p \in B-V \\ f(p) \wedge g(p) \text { if } p \in V \cap B .\end{array}\right.$

It is denoted by $V_{(F, f)} \sqcup_{\mathcal{E}} B_{(\mathfrak{G}, g)}=(V \cup B)_{(F \widetilde{\cup}(\tilde{G}, f \bar{n} g)}$. We shall call this uni-int T-"DFT-soft fuzzy set" over $(U,[0,1])$ as union of "DFT-soft fuzzy set" over $(U,[0,1])$. 
$12 \mid \mathrm{P}$ a g e

\subsection{Theorem}

The extended uni-int "DFT-soft fuzzy set" over $(U,[0,1])$ of two double-framed T-soft fuzzy algebras $((F, f), A)$ and $((\mathfrak{5}, g), A)$ over $(U,[0,1])$ is "DFT-soft fuzzy algebra" over $(U,[0,1])$.

\section{Proof}

Let $u, v \in A$ then we have

$$
\begin{aligned}
(F \widetilde{\cup} \mathfrak{5})(u * v) & =F(u * v) \cup \mathfrak{b}(u * v) \subseteq(F(u) \cup F(v)) \cup(\mathfrak{b}(u) \cup \mathfrak{5}(v)) \\
& =(F(u) \cup(\mathfrak{b}(u)) \cup(F(v) \cup(\mathfrak{5}(v))=(F \widetilde{\cup}(\mathfrak{5})(u) \cup(F \widetilde{\cup} \mathfrak{5})(v), \\
(f \bar{\cap} g)(u * v) & =f(u * v) \wedge g(u * v) \geq(f(u) \wedge f(v)) \wedge(g(u) \wedge g(v)) \\
& =(f(u) \wedge g(u)) \wedge(f(v) \wedge g(v))=(f \bar{\cap} g)(u) \wedge(f \bar{\cap} g)(v) .
\end{aligned}
$$

Hence, $V_{(F, f)} \bar{\square}_{\mathcal{E}} B_{(\mathfrak{G}, g)}$ is a "DFT-soft fuzzy algebra" over $(U,[0,1])$.

\subsection{Definition}

Let $V_{(F, f)}$ and $B_{(\mathfrak{G}, g)}$ are two "DFSS" over $U$. Then extended int-uni "DFT-SS" of $V_{(F, f)}$ and $B_{B_{(\mathfrak{G}, g)}}$ is defined as a "DFSS" $(V \cup B)_{(F \widetilde{\cap} \mathfrak{G}, f \bar{\cup} g)} \operatorname{over}(U,[0,1])$,

Where $F \widetilde{\cap} \mathfrak{5}:(V \cup B) \rightarrow P(U)$ defined by

$$
p \rightarrow\left\{\begin{array}{c}
F(p) \text { if } p \in V-B \\
\mathfrak{5}(p) \text { if } p \in B-V \\
F(p) \cap \mathfrak{5}(p) \text { if } p \in V \cap B
\end{array}\right.
$$

and $f \bar{\cup} g:(V \cup B) \rightarrow[0,1]$ defined by

$$
p \rightarrow\left\{\begin{array}{c}
f(p) \text { if } p \in V-B \\
g(p) \text { if } p \in B-V \\
f(p) \vee g(p) \text { if } p \in V \cap B
\end{array}\right.
$$

It is denoted by $V_{(F, f)} \bar{\Pi}_{\mathcal{E}} B_{(\mathfrak{G}, g)}=(V \cup B)_{(\mathfrak{G} \tilde{n} g, f \bar{\cup} g)}$. We shall call this int-uni "DFT-soft fuzzy set" over $(U,[0,1])$ as intersection of double framed T- soft fuzzy set over $(U,[0,1])$.

\subsection{Theorem}

The extended int-uni "DFT-soft fuzzy set" of two double-framed T-soft fuzzy algebras $((F, f), A)$ and $((\mathfrak{5}, g), A)$ over $(U,[0,1])$ is "DFT-soft fuzzy algebra" over $(U,[0,1])$ if $((F, f), A) \widetilde{\subset}((\mathfrak{G}, g), A)$. 
13 | P a g e

\section{Proof}

Let $u, v \in A$ then we have

$$
\begin{aligned}
(F \widetilde{\cap} \mathfrak{b})(u * v) & =F(u * v) \cap \mathfrak{b}(u * v)=F(u * v) \subseteq F(u) \cup F(v) \\
= & (F(u) \cup \mathfrak{b}(u)) \cap(F(v) \cup \mathfrak{b}(v))=(F \widetilde{\cap} \mathfrak{5})(u) \cup(F \widetilde{\cap} \mathfrak{b})(v), \\
\because & \text { if } \mathrm{A} \subseteq C \text { and } B \subseteq D \text { then } A \cup B=(A \cap C) \cup(B \cap D) . \\
(f \bar{\cup} g)(u * v) & =f(u * v) \vee g(u * v)=f(u * v) \geq f(u) \wedge f(v) \\
\quad & (f(u) \vee g(u)) \wedge(f(v) \vee g(v))=(f \bar{\cup} g)(u) \wedge(f \bar{\cup} g)(v) .
\end{aligned}
$$

Hence, $V_{(F, f)} \overline{\Pi_{\mathcal{E}}} B_{(\mathfrak{b}, g)}$ is a "DFT-soft fuzzy algebra" over $(U,[0,1])$.

\subsection{Theorem}

The extended uni-int "DFT-soft fuzzy set" of two double-framed B-soft fuzzy algebras $((F, f), A)$ and $((\mathfrak{5}, g), A)$ over $(U,[0,1])$ is "DFB-soft fuzzy algebra" over $(U,[0,1])$.

\section{Proof}

Let $u, v \in A$ then we have

$$
\begin{aligned}
(F \widetilde{\cup}(\mathfrak{b})(u * v) & =F(u * v) \cup \mathfrak{b}(u * v) \subseteq(F(u) \cup F(v)) \cup(\mathfrak{b}(u) \cup(\mathfrak{b}(v)) \\
& =(F(u) \cup \mathfrak{b}(u)) \cup(F(v) \cup(\mathfrak{b}(v))=(F \widetilde{\cup} \mathfrak{b})(u) \cup(F \widetilde{\cup} \mathfrak{5})(v), \\
(f \bar{\cap} g)(u * v) & =f(u * v) \wedge g(u * v) \geq(f(u) . f(v)) \wedge(g(u) \cdot g(v)) \ldots \ldots(\mathrm{a})
\end{aligned}
$$

Case (1)

If $(f(u) \cdot f(v)) \wedge(g(u) \cdot g(v))=(f(u) \cdot f(v))$ then (a) becomes

$(f \bar{\cap} g)(u * v) \geq(f(u) \cdot f(v)) \geq(f(u) \wedge g(u)) \cdot(f(v) \wedge g(v))=(f \bar{\cap} g)(u) \cdot(f \bar{\cap} g)(v)$

Implies $(f \bar{\cap} g)(u * v) \geq(f \bar{\cap} g)(u) \cdot(f \bar{\cap} g)(v)$.

Case (2)

If $(f(u) \cdot f(v)) \wedge(g(u) \cdot g(v))=(g(u) \cdot g(v))$ then (a) becomes 
14| P a g e

$(f \bar{\cap} g)(u * v) \geq(g(u) \cdot g(v)) \geq(f(u) \wedge g(u)) \cdot(f(v) \wedge g(v))=(f \bar{\cap} g)(u) \cdot(f \bar{\cap} g)(v)$

Implies $(f \bar{\cap} g)(u * v) \geq(f \bar{\cap} g)(u) .(f \bar{\cap} g)(v)$.

Hence, proof is complete.

Hence, $V_{(F, f)} \bar{\sqcup}_{\mathcal{\varepsilon}} B_{(\mathfrak{b}, g)}$ is a "DFB-soft fuzzy algebra" over $(U,[0,1])$.

\subsection{Theorem}

The extended int-uni "DFT-soft fuzzy set" of two double-framed B-soft fuzzy algebras $((F, f), A)$ and $((\mathfrak{5}, g), A)$ over $(U,[0,1])$ is "DFB-soft fuzzy algebra" over $(U,[0,1])$ if $((F, f), A) \widetilde{\subset}((\mathfrak{b}, g), A)$.

\section{Proof}

Let $u, v \in A$ then we have

$$
\begin{aligned}
&(F \widetilde{\cap}(\mathfrak{5})(u * v)= F(u * v) \cap \mathfrak{b}(u * v)=F(u * v) \subseteq F(u) \cup F(v) \\
&=(F(u) \cup \mathfrak{b}(u)) \cap(F(v) \cup(\mathfrak{G}(v))=(F \widetilde{\cap} \mathfrak{b})(u) \cup(F \widetilde{\cap}(\mathfrak{b})(v), \\
& \because \text { if } A \subseteq C \text { and } B \subseteq D \text { then } A \cup B=(A \cap C) \cup(B \cap D) . \\
&(f \bar{\cup} g)(u * v)= f(u * v) \vee g(u * v)=g(u * v) \geq(g(u) \wedge g(v)) \\
&=(f(u) \vee g(u)) .(f(v) \vee g(v))=(f \bar{\cap} g)(u) \wedge(f \bar{\cap} g)(v) .
\end{aligned}
$$

Hence, $V_{(F, f)} \overline{\Pi_{\mathcal{E}}} B_{(\mathfrak{b}, g)}$ is a "DFB-soft fuzzy algebra" over $(U,[0,1])$.

For a "DFT-soft fuzzy set" $((F, f), A)$, we defined $\psi-$ exclusive and $\alpha-$ cut respectively as follows, where $\psi \subseteq U$ and $\alpha \in[0,1]$

$$
\begin{aligned}
& \psi-\text { exclusive }=\{u \in A \mid \psi \supseteq F(u)\}, \\
& \alpha-c u t=\{u \in A \mid \alpha \leq f(u)\}
\end{aligned}
$$

and denoted as $e_{A}(F ; \psi)$ and $f_{\alpha}$, respectively.

The set $\operatorname{DFT}_{\mathrm{A}}(F, f)_{(\psi, \alpha)}=\{u \in A \mid \psi \supseteq F(u), \alpha \leq f(u)\}$ is called double framed including set.

\subsection{Theorem}

For a "DFT-soft fuzzy set" $((F, f), \delta)$ over $(U,[0,1])$, the following are equivalent

(1) $((F, f), \delta)$ is double framed soft fuzzy algebra over $(U,[0,1])$, 
$15 \mid \mathrm{P}$ a g e

(2) for every $\psi \subseteq U$ and $\beta \in[0,1]$ with $\psi \in \operatorname{Im}(F)$ and $\alpha \in \operatorname{Im}(f)$ then $\psi-$ exclusive set and $\alpha-$ cut of $((F, f), \delta)$ are sub algebras of $\delta$.

Proof

$(1) \Rightarrow(2)$

Consider $((F, f), \delta)$ is "DFT-soft fuzzy algebra" over $(U,[0,1])$. Let $u, v \in \delta$ be such that $u, v \in e_{\delta}(F ; \psi)$ and $u, v \in f_{\alpha}$ for every $\psi \subseteq U$ and $\alpha \in[0,1]$ respectively, with $\psi \in \operatorname{Im}(F)$ and $\alpha \in \operatorname{Im}(f)$. Then by definition of $((F, f), \delta)$ such that

$F(u * v) \subseteq F(u) \cup F(v) \subseteq \psi, \quad g(u * v) \geq g(u) \wedge g(v) \geq \alpha$

Implies $u * v \in e_{\delta}(F ; \psi)$ and $u * v \in f_{\alpha}$.

Hence, $e_{\delta}(F ; \psi)$ and $f_{\alpha}$ are subalgebras of $\delta$.

$(2) \Rightarrow(1)$, Suppose that $(2)$ is valid to prove $((F, f), \delta)$ is double framed soft fuzzy algebra $\operatorname{over}(U,[0,1])$. Let $u, v \in \delta$ be such that $F(u)=\psi_{u}, F(v)=\psi_{v}$ and $f(u)=\alpha_{u}, f(v)=$ $\alpha_{v}$. By taking $\psi=\psi_{u} \cup \psi_{v}$ and $\alpha=\alpha_{u} \wedge \alpha_{v}$. Then $u * v \in e_{\delta}(F ; \psi)$ and $u * v \in f_{\alpha}$.

As $e_{\delta}(F ; \psi)$ and $f_{\alpha}$ are double framed T-soft fuzzy subalgebra so

$F(u * v) \subseteq \psi=\psi_{u} \cup \psi_{v}=F(u) \cup F(v), \quad g(u * v) \geq \alpha=\alpha_{u} \wedge \alpha_{v}=g(u) \wedge g(v)$.

Hence, $((F, f), \delta)$ is "DFT-soft fuzzy algebra" over $(U,[0,1])$.

\subsection{Corollary}

If $((F, f), \delta)$ is a "DFT-soft fuzzy algebra" over $(U,[0,1])$ then double framed including set of $((F, f), \delta)$ is subalgebra $X$.

\subsection{Definition}

Let $((F, f), \delta)$ be a "DFT-soft fuzzy set" over $(U,[0,1])$. Then "DFT-soft fuzzy set" $\left(\left(F^{*}, f^{*}\right), \delta\right)$ over $(U,[0,1])$ is defined by

$F^{*}: \delta \rightarrow P(U) p \rightarrow\left\{\begin{array}{c}F(p) p \in e_{\delta}(F ; \psi) \\ M \text { otherwise }\end{array}\right.$

$f^{*}: \delta \rightarrow[0,1] \quad p \rightarrow \begin{cases}f(p) & \text { if } p \in f_{\alpha} \\ \gamma & \text { therwise }\end{cases}$

Where $\psi, M \subseteq U, \alpha, \gamma \in[0,1]$ and $F(p) \subset M, f(p)>\gamma$.

\subsection{Theorem}

If $((F, f), \delta)$ is a "DFT-soft fuzzy algebra" over $(U,[0,1])$. Then prove that $\left(\left(F^{*}, f^{*}\right), \delta\right)$ over $(U,[0,1])$ is also a "DFT-soft fuzzy algebra". 
$16 \mid \mathrm{P}$ a g e

\section{Proof}

Let $((F, f), \delta)$ be a "DFT-soft fuzzy algebra" over $(U,[0,1])$, to prove $\left(\left(F^{*}, f^{*}\right), \delta\right)$ is also a "DFT-soft fuzzy algebra" over $(U,[0,1])$.

As $((F, f), \delta)$ be a "DFT-soft fuzzy algebra" over $(U,[0,1])$ so for every $\psi \subseteq U$ and $\alpha \in$ $[0,1], e_{\delta}(F ; \psi)$ and $f_{\alpha}$ are sub algebras with $\psi \in \operatorname{Im}(F)$ and $\alpha \in \operatorname{Im}(f)$.

Let $u, v \in \delta$ if $u, v \in e_{\delta}(F ; \psi)$ then $u * v \in e_{\delta}(F ; \psi)$.

Thus, $F^{*}(u * v)=F(u * v) \subseteq F(u) \cup F(v)=F^{*}(u) \cup F^{*}(v)$,

If $u \notin e_{\delta}(F ; \psi)$ or $v \notin e_{\delta}\left(F ; \psi\right.$ then $F^{*}(u)=M$ or $F^{*}(v)=M$,

Thus, $F^{*}(u * v) \subseteq M=F^{*}(u) \cup F^{*}(v) \because F(p) \subset M$ if $p \notin e_{\delta}(F ; \psi)$.

Now, if $u, v \in i_{\delta}(f ; \alpha)=f_{\alpha}$ then $u * v \in f_{\alpha}$.

Thus, $f^{*}(u * v)=f(u * v) \geq f(u) \wedge f(v)=f^{*}(u) \wedge f^{*}(v)$

Implies, $f^{*}(u * v) \geq f^{*}(u) \wedge f^{*}(v)$.

If $u \notin f_{\alpha}$ or $v \notin f_{\alpha}$ then $f^{*}(u)=\gamma$ or $f^{*}(v)=\gamma$,

Thus,

$f^{*}(u * v)=f(u * v) \geq \gamma=f(u) \wedge f(v)=f^{*}(u) \wedge f^{*}(v) \because f(p)>\gamma$ if $p \notin f_{\alpha}$.

\subsection{Definition}

Let $((F, f), A)$ and $((F, f), B)$ be "DFT-soft fuzzy set" over $(U,[0,1])$. Then their product is defined as

$$
\begin{aligned}
& F_{A \vee B}: A \times B \rightarrow P(U), \\
& f_{A \wedge B}: A \times B \rightarrow[0,1]
\end{aligned}
$$

Such that $(x, y) \rightarrow\left\{\begin{array}{l}F(x) \cup F(y) \\ f(x) \wedge f(y)\end{array}\right.$

And denoted by $\left(\left(F_{\vee}, f_{\wedge}\right), A \times B\right)$.

\subsection{Theorem}

Let $((F, f), A)$ and $((F, f), B)$ be double framed T-soft fuzzy algebras over $(U,[0,1])$. Then prove that $\left(\left(F_{\mathrm{V}}, f_{\wedge}\right), A \times B\right)$ is also a "DFT-soft fuzzy algebra" over $(U,[0,1])$.

Proof

We know that $(A \times B, \circledast,(\theta, \theta)$ is also a $\mathrm{BCK} / \mathrm{BCI}$ algebra. Then we only prove that $\left(\left(F_{\mathrm{V}}, f_{\wedge}\right), A \times B\right)$ is a "DFT-soft fuzzy algebra" over $(U,[0,1])$. 
17 | P a g e

Let $(x, y),(u, v) \in A \times B$, we have

$$
\begin{gathered}
F_{A \vee B}((x, y) \circledast(u, v))=F_{A \vee B}(x * u, y * v) \\
=F(x * u) \cup F(y * v) \subseteq(F(x) \cup F(u)) \cup(F(y) \cup F(v)) \\
=(F(x) \cup F(y)) \cup(F(u) \cup F(v)) \\
=F_{A \vee B}(x, u) \cup F_{A \vee B}(y, v) . \\
f_{A \wedge B}((x, y) \circledast(u, v))=f_{A \wedge B}(x * u, y * v)=f(x * u) \wedge f(y * v) \\
\geq(f(x) \wedge f(u)) \wedge(f(y) \wedge f(v)) \\
=(f(x) \wedge f(y)) \wedge(f(u) \wedge f(v)) \\
= \\
=f_{A \wedge B}(x, y) \wedge f_{A \wedge B}(u, v) .
\end{gathered}
$$

Hence, $\left(\left(F_{\mathrm{V}}, f_{\wedge}\right), A \times B\right)$ is a "DFT-soft fuzzy algebra" over $(U,[0,1])$.

\subsection{Theorem}

Let $((F, f), A)$ and $((F, f), B)$ be double framed T-soft fuzzy algebras over $(U,[0,1])$. Then prove that $\left(\left(F_{\mathrm{V}}, f_{\wedge}\right), A \times B\right)$ is also a "DFB-soft fuzzy algebra" over $(U,[0,1])$.

Proof

We similar to above theorem by using definition of double framed T-soft fuzzy algebra and previous theorem.

\section{Conclusion:}

We defined the concept of "DFT-soft fuzzy set" which is combination of double framed T soft fuzzy set and introduced their notions. Further, proved that extended uni-int of "DFT-soft fuzzy set" is also double framed T-soft fuzzy algebra and "DFB-soft fuzzy algebra" but converse is not true because, we proved that each double framed $\mathrm{T}$ soft fuzzy algebra is also "DFB-soft fuzzy algebra" but converse is not true. And we also discussed the product of double framed T-soft fuzzy algebra and "DFB-soft fuzzy algebra".

Further, we will apply this concept in different algebraic structure and discussed its operations.

\section{Bibliography}

[1] Phuong, N. H., \& Kreinovich, V. (2001). Fuzzy logic and its applications in medicine. International journal of medical informatics, 62(2-3), 165-173.

[2] Zadeh, L. A. (1996). Fuzzy sets. In Fuzzy Sets, Fuzzy Logic, And Fuzzy Systems: Selected Papers by Lotfi A Zadeh (pp. 394-432). 
18 | P a g e

[3] Molodtsov, D. (1999). Soft set theory-first results. Computers \& Mathematics with Applications, 37(4-5), 19-31.

[4] Maji, P. K., Roy, A. R., \& Biswas, R. (2002). An application of soft sets in a decision making problem. Computers \& Mathematics with Applications, 44(8-9), 1077-1083.

[5] Maji, P. K., Biswas, R., \& Roy, A. (2003). Soft set theory. Computers \& Mathematics with Applications, 45(4-5), 555-562.

[6] Aktaş, H., \& Çağman, N. (2007). Soft sets and soft groups. Information sciences, 177(13), 2726-2735.

[7] Ali, M. I., Feng, F., Liu, X., Min, W. K., \& Shabir, M. (2009). On some new operations in soft set theory. Computers \& Mathematics with Applications, 57(9), 1547-1553.

[8] Huang, Y. (2006). BCI-algebra. Elsevier.

[9] Meng, J., \& Jun, Y. B. (1994). BCK-algebras. Kyung Moon Sa Company.

[10] Jun, Y. B., Kang, M. S., \& Lee, K. J. (2013). Intersectional soft sets and applications to BCK/BCI-algebras. Communications of the Korean Mathematical Society, 28(1), 11-24.

[11] Jun, Y. B. (2013). Union-soft sets with applications in BCK/BCI-algebras. Bulletin of the Korean Mathematical Society, 50(6), 1937-1956.

[12] Jun, Y. B., \& Park, C. H. (2008). Applications of soft sets in ideal theory of BCK/BCIalgebras. Information Sciences, 178(11), 2466-2475.

[13] Jun, Y. B., \& Ahn, S. S. (2012). Double-framed soft sets with applications in BCK/BCIalgebras. Journal of Applied Mathematics, 2012.

[14] Naz. M., (2015). Some studies on algebraic structure of soft sets. P.hd Dissertion Quaidi-Azam University Islamabad, Pakistan.

[15] Hadipour, A. R. (2014). Double-framed Soft BF-algebras. Indian Journal of Science and Technology, 7(4), 491-496.

[16] Cho, Y. U., Lee, K. J., \& Jun, Y. B. (2015). A study on double-framed soft nearrings. Applied Mathematical Sciences, 9(18), 867-873.

[17] Shabir, M. \& Khan, S. (2016). Double framed soft topological space, Annals of Fuzzy Mathematics and Informations, $\mathrm{x}(\mathrm{x}), 1-\mathrm{xx}$.

[18] Jun, Y. B., Lee, K. J., \& Roh, E. H. (2012). Intersectional soft BCK/BCI-ideals. Annals of Fuzzy Mathematics and Informatics, 4(1), 1-7.

[19] Mahmood, T., Ullah, M., Khan, M. B., \& Ullah, K. (2017). On Twisted Soft Ideal of Soft Ordered Semigroups. JOURNAL OF INFORMATION COMMUNICATION TECHNOLOGIES AND ROBOTICS APPLICATIONS (JICTRA).(Formally known as Journal of Computer Science of NICE). ISSN\# 2226-3683, 8, 74-85. 
19 | P a g e

[20] Acar, U., Koyuncu, F., \& Tanay, B. (2010). Soft sets and soft rings. Computers \& Mathematics with Applications, 59(11), 3458-3463.

[21] Bal, M.; Shalla, M.M.; Olgun, N. Neutrosophic Triplet Cosets and Quotient Groups. Symmetry 2018, 10, 126.

[22] Sezer, A. S., \& Atagün, A. O. (2016). A New Kind of Vector Space: Soft Vector Space. Southeast Asian Bulletin of Mathematics, 40(5), 753-770.

[23] Ali, M. I., Shabir, M., \& Feng, F. (2017). Representation of graphs based on neighborhoods and soft sets. International Journal of Machine Learning and Cybernetics, 8(5), 1525-1535.

[24] Jun, Y. B., Kim, H. S., \& Neggers, J. (2009). Pseudo d-algebras. Information Sciences, 179(11), 1751-1759.

[25] Jun, Y. B., Lee, K. J., \& Khan, A. (2010). Soft ordered semigroups. Mathematical Logic Quarterly, 56(1), 42-50.

[26] Jun, Y. B., Lee, K. J., \& Park, C. H. (2009). Soft set theory applied to ideals in dalgebras. Computers \& Mathematics with Applications, 57(3), 367-378.

[27] Jun, Y. B., Lee, K. J., \& Zhan, J. (2009). Soft p-ideals of soft BCI-algebras. Computers \& Mathematics with Applications, 58(10), 2060-2068.

[28] Khan, A., Izhar, M., \& Khalaf, M. M. (2017). Double-framed soft LAsemigroups. Journal of Intelligent \& Fuzzy Systems, 33(6), 3339-3353.

[29] Hussain, R. J., Sampath, K., \& Jayaraman, P. (2017). Applications of Double-Framed Soft Ideal Structures over Gamma Near-Rings. Global Journal of Pure and Applied Mathematics, 13(9), 5101-5113.

[30] Khan, A., Izhar, M., \& Sezign, A. (2017). Characterizations of Abel Grassmann's Groupoids by the Properties of Their Double-Framed Soft Ideals. International Journal of Analysis and Applications, 15(1), 62-74. 